\title{
AN EXPERIMENTAL CAR-BORNE SAR SYSTEM: MEASUREMENT SETUP AND POSITIONING ERROR ANALYSIS
}

\author{
Roberto Coscione ${ }^{1}$, Irena Hajnsek ${ }^{1,2}$, Othmar Frey ${ }^{1,3}$ \\ ${ }^{1}$ Earth Observation and Remote Sensing, ETH Zurich, Switzerland \\ ${ }^{2}$ Microwaves and Radar Institute, German Aerospace Center - DLR, Oberpfaffenhofen, Germany \\ ${ }^{3}$ Gamma Remote Sensing AG, Gümligen, Switzerland
}

\begin{abstract}
Repeat-pass differential SAR interferometry (DInSAR) using spaceborne SAR data or stationary terrestrial radar data is an established technique to measure surface displacements. However, repeat-pass DInSAR from agile platforms (airborne/car-borne) is challenging due to residual motion errors. This is particularly true for high-frequency radar where motion errors of few millimeters represent a non-negligible fraction of the wavelength. In this paper, an experimental car-borne SAR system is presented. Such a system is complementary to the existing solutions (namely spaceborne, airborne, and terrestrial systems) in terms of geometry of acquisition, and flexibility in the selection of temporal baselines and location of the acquisitions. To meet the need of consistent and precise trajectory information, proper postprocessing procedure must be applied to the raw positioning data collected from the inertial navigation system (INS) and the global positioning system (GNSS). A viable procedure is here presented and first results discussed.
\end{abstract}

Index Terms - DInSAR, car-borne, INS, GNSS

\section{INTRODUCTION}

Monitoring surface changes is of primary importance for hazard management and for many practical and scientific purposes. Repeat-pass differential SAR interferometry (DInSAR) is specifically suitable for measuring surface deformations, although its viability is limited by the decorrelation behavior of the imaged surface. This is particularly critical over vegetated areas where a complete decorrelation often occurs even when the radar acquisitions are separated by a short time-span. Areas where changes occur at a fast rate should be monitored using relatively short temporal baselines between the acquisitions, so that it is possible to track such changes and avoid complete decorrelation. Few options are currently available for monitoring specific regions of interest with temporal baselines adaptable to site-specific needs, namely airborne and terrestrial SAR systems. Airborne systems typically have a high cost per mission, which limits the possibility to acquire large data stacks (required for advanced DInSAR processing) and to perform long-term measurements. Ground-based SAR systems are constrained by the use of high frequency radar to achieve a good azimuth resolution and to maintain an overall compact dimension of the system [1]. However, high frequency bands like the commonly used Ku-band are very sensitive to intra-resolution-cell movements in the imaged area and are prone to exhibit strong temporal decorrelation [2]. In this paper, an experimental car-borne SAR system suitable for repeat-pass DInSAR measurements is presented. Compared to airborne or spaceborne SAR, such a system is a complementary tool in terms of geometry of acquisition and flexibility in the choice of the location and the temporal baseline. Furthermore, the length of the synthetic aperture is not constrained by the length of a rail, as in the case of terrestrial SAR systems. This allows the use of lower-frequency electromagnetic signals, which are better suited to monitor for example natural surfaces, and to still approach the full azimuth resolution of the SAR. Like for the airborne case, the proposed system is subject to unkown residual motion errors due to inaccuracies in the measured trajectory. Particularly, in view of repeat-pass interferometric measurements a highly accurate sensor positioning is crucial. While a first car-borne SAR demonstration has been carried out using only carrier-phase-based differential GNSS measurements for positioning [3, 4], our experimental car-borne SAR system is now equipped with a navigation-grade inertial navigation system (INS) system. In this paper, the positioning performances are discussed in this context.

\section{SYSTEM DESCRIPTION}

The experimental car-borne SAR system is shown in Fig. 1. It is currently composed of:

- a Ku-band FMCW radar system: a modified variant of the Gamma Portable Radar Inteferometer (GPRI, see also $[3,5])$ with a nominal frequency of $17.2 \mathrm{GHz}$ and $200 \mathrm{MHz}$ of chirp bandwidth $(0.75 \mathrm{~m}$ of range pixel spacing)

- $3 \mathrm{Ku}$-band V-polarized horn antennas (1 transmitting and 2 receiving) and a GNSS antenna 


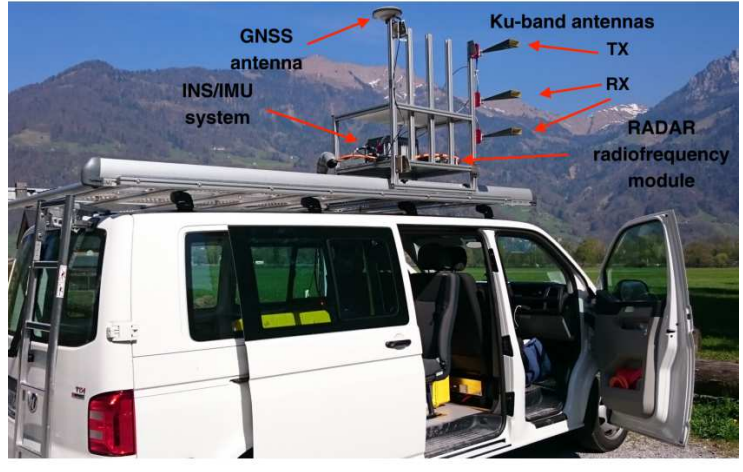

Fig. 1: Ku-band car-borne SAR system

- an inertial navigation system (INS, model iNAV-RQH10018 provided by iMAR Navigation GbmH) with integrated 3-axis quartz-based servo accelerometers, 3axis ring-laser gyroscopes, and a GNSS receiver. Free inertial position accuracy below $0.6 \mathrm{~nm} / \mathrm{h}$ (nautical miles per hour), data rate up to $300 \mathrm{~Hz}$.

Although the goal is the use a lower frequency band, suitable for long-term repeat-pass DInSAR measurements, the higher sensitivity of the Ku-band system to residual motion errors allows to test the performance of the post-processing procedure applied to the INS/GNSS positioning data. The terms free-run or free inertial position refer to the solution calculated without GNSS aiding. Although better accuracy is achieved by combining inertial and GNSS data, the free inertial accuracy is still relevant for SAR applications, as it will be clarified later.

\section{NAVIGATION MEASUREMENT SETUP}

The INS/GNSS and radar measurements are carried out as follows. At the beginning of the campaign, a standstill INS/GNSS acquisition of at least 10 minutes is conducted. This permits to perform leveling and North-seeking procedures during post-processing to correctly initialize INS attitude parameters. SAR acquisitions are right-looking and the acquisitions length are typically between 30 and $60 \mathrm{sec}-$ onds or more, depending on the velocity of the platform and the distance of the target scene.

\section{INERTIAL PROCESSING}

Position errors dramatically affect focusing and interferometric performances and need to be compensated in some way. INS systems are affected by biases in the gyroscopes and the accelerometers. These cause a position drift that increases quadratically with time, due to the double integration required to calculate the position from the acceleration. Furthermore, such biases are not constant over time but change for example with the temperature (e.g. due to internal heating of the system). A common approach to correct such a drift is to combine INS and GNSS data through Kalman filtering. The rationale is that while INSs are accurate on short time-span, GNSS systems provide accurate data over long time-span and are not affected by position drift. GNSS data, which is typically available at a rate of $1 \mathrm{~Hz}$, is used to periodically correct the INS position drift. However, such correction produces a sawtooth-like signal that causes phase artifacts in the focused radar image. A filtering can be applied in order to attenuate this effect, but position offset and/or undulation would remain in the output signal. To avoid this unwanted effect, an approach exploiting the high-performances of the available INS can be used. The processing procedure is summarized as follows. The GNSS data is processed using a single- or dualfrequency carrier-phase differential GNSS technique known as Real-Time Kinematic (RTK). The INS/GNSS data fusion is then performed with an extended Kalman filter followed by a Rauch-Tung-Striebel (RTS) smoother [6], which is known for decreasing the estimation error when used in cascade with a Kalman filter [7]. Afterwards, a free inertial solution, i.e. without GNSS/RTK aiding, is extracted from the navigation data in correspondence to the start and end times of the radar acquisitions, using the RTS output solution as starting point. In this way, the inertial drift starts accumulating only from the beginning of every radar acquisition until its end. To further reduce the inertial drift, a polynomial function fitting the difference between the "windowed" free-run position and the RTS output is calculated and then subtracted from the former. Finally, a low-pass Butterworth filter of the 5th order is applied to the result in order to remove high frequency components caused by residual noise and vibrational contributions from the car and the roughness of the street. At this stage, the estimated position is the one of the reference point situated inside the INS chassis. To supply this data to the focusing algorithm, the position of the antennas phase center (APC) during the transmission of the RF pulses needs to be calculated. This is done by measuring the lever-arms from the INS reference point to the APC and by applying a rigid body transformation taking into account the attitude information from the RTS output. Finally, the position of the APC is resampled to the radar pulse repetition frequency.

\section{RTK AND RTS SENSITIVITY ANALYSIS}

As previously mentioned, RTK processing is a basic step for the estimation of the trajectory of the car (more generically called rover in the context of GNSS processing).

Switzerland has a network of more than 30 stable and continuously operating GNSS base-stations (Automated GNSS Network for Switzerland, AGNES), supporting carrier-phasebased static or kinematic differential GNSS processing. Ideally, one could rely on one of these permanent GNSS stations 
or on a derived virtual GNSS stations for the INS/GNSS postprocessing of the navigation data. In the following we analyse the performance of the positioning solution for different GNSS baselines as they may occur in a DInSAR measurement scenario. We tested the robustness of the RTK solutions to variation of the baseline and the effect of the RTKs trajectory on the combined RTS one.

A test measurement has been performed close to the ETH Hönggerberg campus, where an AGNES base-station (called ETH) is situated $500 \mathrm{~m}$ apart from the rover. Separate RTK solutions have been processed with respect to the ETH basestation and a second one (FRI3) having a baseline to the rover of $30 \mathrm{Km}$ circa. In the case of FRI3, L1 and L2 carrier signals have been used to allow for ionospheric path delay correction, at the cost of increased variance. The short baseline in the case of the ETH base-station allows to perform RTK processing using only the L1 signal, because ionospheric and tropospheric path delays at the rover and the base-station mutually cancel out during differential processing. It is thus expected a reduced uncertainty of the GNSS positioning solution with respect to longer baselines.

In the context of repeat-pass DInSAR measurements, position errors should be kept to a minimum. When the rover is not close to a base-station, a possible data acquisition scheme aimed at reducing position estimation errors is to use a fixed GNSS receiver close to the rover and in open sky. The position of such fixed non-permanent receiver can be calculated using a static differential GNSS technique with respect to the closest AGNES base-station. The rover position can be finally calculated via RTK processing considering the fixed receiver as base-station and using only the L1 carrier. Clearly, from an operational point of view, it would be beneficial to rely only on the permanent GNSS network instead of installing a separate reference station close to the SAR platform. In the following, we compare the two cases.

Altitude errors usually represent the worst case in the context of RTK position estimation. Fig. 2a shows the altitude difference of the RTK solutions as well as of the RTS ones. The two RTK solutions have a mean difference of circa $7 \mathrm{~cm}$. During standstill sessions (occurring at different positions) a constant offset can be measured thanks to the reduced uncertainty of the position estimation. As shown in Fig. 2b, the variability visible during kinematic sessions is strictly correlated to the drop in the number of satellites visible from the rover and the base-stations, which negatively affects the precision dilution of position (PDOP) and the standard deviation of the RTK solution. This is caused by satellites occultation occurring between GPS time $489500 \mathrm{~s}$ and $490000 \mathrm{~s}$ and after each radar acquisition (all of them starting and ending approximately at the same positions) due to trees and man-made structures. As expected, the RTS solutions show more contained and smoother variations.

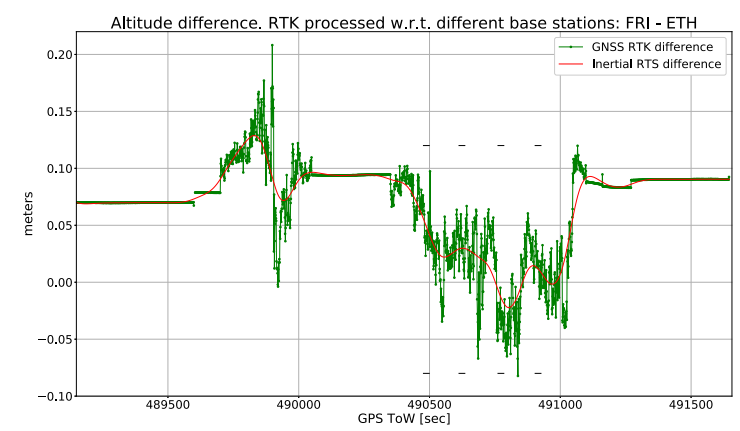

(a) In green: difference of RTK altitude processed w.r.t. ETH and FRI3 AGNES base-stations. In red: difference in altitude of the RTS output obtained with said RTKs as input. Black lines indicate the time windows during which radar acquisitions have been performed

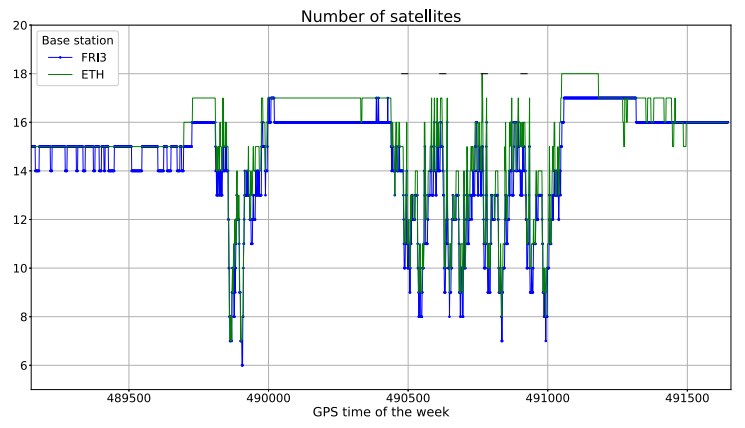

(b) Number of satellites visible from the rover and the base-station

Fig. 2

\section{INS SOLUTION: ERROR ANALYSIS}

Fig. 3a shows the inertial drift affecting the windowed freerun solution. This has been calculated as the difference between the windowed free-run solution and the RTS output. Because the free-run solution is calculated using the RTS output as starting point, at time $\mathrm{t}=0$ there is no difference between the two. Afterwards, the accumulation of the inertial drift becomes apparent: after 40 seconds there is a difference of $14 \mathrm{~cm}$ circa on the $y$-component and $4 \mathrm{~cm}$ circa on the $\mathrm{z}$ component. At Ku-band (wavelength $1.75 \mathrm{~cm}$ ), such errors would determine an uncertainty of multiple phase cycles on the received echoes. Fig. $3 b$ shows the difference between the two solutions after applying the detrending procedure to the free-run data, as described in Section 4. The improvement is evident: the residual error is mostly confined below $0.25 \mathrm{~mm}$. The points where the residual error worsen are correlated with irregularities of the road surface. 


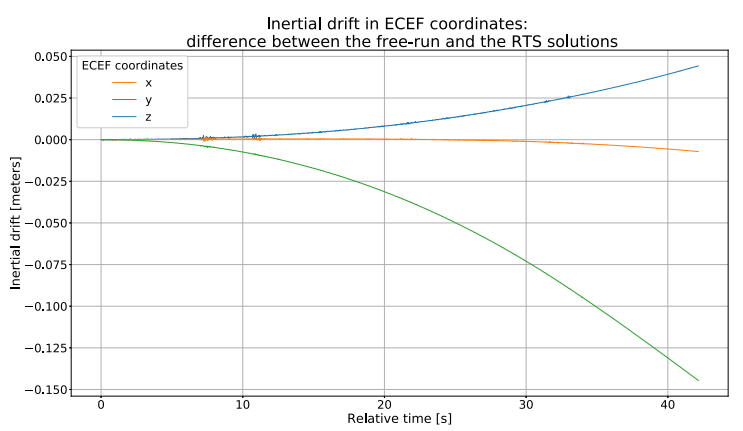

(a) Inertial drift in ECEF coordinates calculated as the difference between the windowed free-run solution and the RTS output

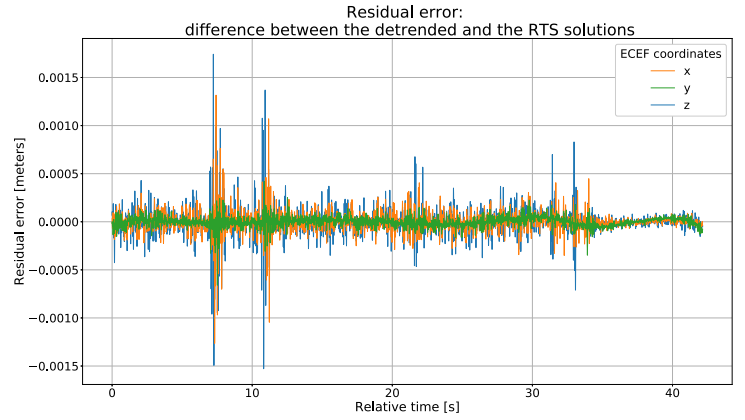

(b) Residual error between the windowed free-run solution after detrending and the RTS output

Fig. 3

\section{CONCLUSIONS}

In this paper, an experimental car-borne SAR system has been presented. The system configuration and the measurement setup have been described. Repeat-pass DInSAR measurements require precise and consistent position estimation from pass to pass. Positioning aspects for a car-borne SAR system along with sources of error and a viable inertial processing sequence have been described. First results show the suitability of the proposed procedure for reducing drift errors due to biases in the gyroscope and the accelerometers of the INS.

\section{REFERENCES}

[1] Rafael Caduff, Fritz Schlunegger, Andrew Kos, and Andreas Wiesmann, "A review of terrestrial radar interferometry for measuring surface change in the geosciences," Earth Surface Processes and Landforms, vol. 40, no. 2, pp. 208-228, 2015.

[2] Howard Zebker and John Villasenor, "Decorrelation in interferometric radar echoes," IEEE Transactions on Geoscience and Remote Sensing, vol. 30, no. 5, pp. 950 959, Sep 1992.

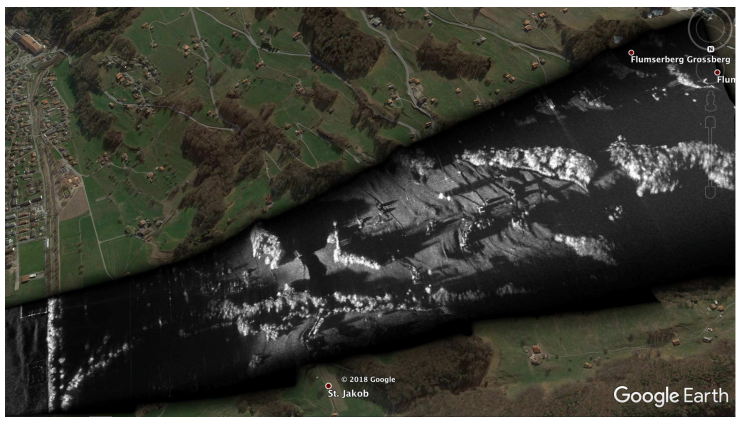

Fig. 4: Focused and geocoded radar image acquired from the car platform in the area of Flums, Switzerland. Azimuth focusing: time domain back-projection $[8,9]$

[3] Othmar Frey, Charles L. Werner, Urs Wegmuller, Andreas Wiesmann, Daniel Henke, and Christophe Magnard, "A car-borne SAR and InSAR experiment," in Proc. IEEE Int. Geosci. Remote Sens. Symp., July 2013 , pp. 93-96.

[4] Othmar Frey, Charles L. Werner, and Urs Wegmuller, "GPU-based parallelized time-domain back-projection processing for agile SAR platforms," in Proc. IEEE Int. Geosci. Remote Sens. Symp., July 2014, pp. 1132-1135.

[5] Charles L. Werner, Tazio Strozzi, Andreas Wiesmann, and Urs Wegmuller, "A real-aperture radar for groundbased differential interferometry," in Proc. IEEE Int. Geosci. Remote Sens. Symp., July 2008, vol. 3, pp. 210213.

[6] H. E. Rauch, C. T. Striebel, and F. Tung, "Maximum likelihood estimates of linear dynamic systems," AIAA Journal, vol. 3, no. 8, pp. 1445-1450, Aug 1965.

[7] H. Liu, S. Nassar, and N. El-Sheimy, "Two-filter smoothing for accurate ins/gps land-vehicle navigation in urban centers," IEEE Transactions on Vehicular Technology, vol. 59, no. 9, pp. 4256-4267, Nov 2010.

[8] Othmar Frey, Erich Meier, and Daniel Nüesch, "Processing SAR data of rugged terrain by time-domain backprojection," in SPIE Vol. 5980: SAR Image Analysis, Modeling, and Techniques X, 2005.

[9] Othmar Frey, Christophe Magnard, Maurice Rüegg, and Erich Meier, "Focusing of airborne synthetic aperture radar data from highly nonlinear flight tracks," IEEE Trans. Geosci. Remote Sens., vol. 47, no. 6, pp. 18441858, June 2009. 